\title{
Aproximación metodológica al estudio de la dirección orquestal desde el punto de vista de la obra musical
}

\section{Methodological approach to the study of orchestral conducting from the point of view of the musical work}

\author{
Marta Vela González \\ Universidad Internacional de La Rioja \\ marta.vela@unir.net \\ ORCID iD: http://orcid.org/0000-0002-5700-6767
}

\section{RESUMEN}

Este artículo pretende reflexionar sobre el estudio de la dirección orquestal desde el punto de visto del estudio y la reflexión de la obra musical. Frente a la manifiesta escasez de bibliografía sobre este tema tan específico, se pretende ofrecer una amplia visión diacrónica, aportada por grandes figuras de la dirección orquestal, desde los inicios, con la profesionalización de la figura del director -Mendelsshon, Berlioz, Wagner-, la época dorada, en la primera mitad del siglo XX -Mahler, Walter, Furtwängler, Weintgartner, Swarowsky, Scherchen, Ansermet-, el período de posguerra y la segunda mitad del siglo XX -Muti, Karajan, Solti, Bernstein, Abbado- y la época actual -Thielemann, Rattle, Ros-Marbà-. La aproximación de los grandes intérpretes a la obra parte de un estudio profundo de la obra musical a partir del análisis que parte de un punto claro y lleno de interés en el proceso de estudio: la imagen previa del sonido, estrategia indispensable y anterior al ensayo, que puede extrapolarse al piano y al resto de los instrumentos y al canto.

Palabras clave: música, dirección orquestal, piano, enseñanza, análisis musical. 


\title{
MARTA VELA GONZÁLEZ
}

\begin{abstract}
This article aims to reflect on the study of orchestral conducting from the point of view of studying and reflecting on the musical work. Faced with the manifest scarcity of bibliography on this very specific topic, it is intended to offer a broad diachronic vision, contributed by great figures of orchestral conducting, from the beginning, with the professionalization of the figure of the conductor-Mendelsshon, Berlioz, Wagner-, the golden age, in the first half of the 20th century -Mahler, Walter, Furtwängler, Weintgartner, Swarowsky, Scherchen, Ansermet-, the post-war period and the second half of the 20th century -Muti, Karajan, Solti, Bernstein, Abbado- and the present time -Thielemann, Rattle, Ros-Marbà-. The approach of the great interpreters to the work starts from a deep study of the musical work from the analysis that starts from a clear point full of interest in the study process: the previous image of the sound, an indispensable and previous strategy to rehearsal, which can be extrapolated to the piano and the rest of the instruments and to singing.
\end{abstract}

Key Words: music, orchestral conducting, piano, teaching, musical analysis.

Vela González, M. (2022). Aproximación metodológica al estudio de la dirección orquestal desde el punto de vista de la obra musical. Cuadernos de Investigación Musical, (14), pp. 223-249.

\section{INTRODUCCIÓN}

Todo proceso de aprendizaje parte de la curiosidad del alumno, de su deseo de saber $y$ de penetrar constantemente en un campo desconocido: "One of man's natural attributes is curiosity. He has been asking the question «Why?» for so long that it never occurs to him to consider why he does so although he may often risk disillusionment" (Walker, 1962, p. 13).

Así pues, como recordaba Schoenberg, el arte requiere el ansia de saber:

El impulso más noble de todos, el impulso de conocer nos impone el deber de buscar. Y una doctrina errónea basada en una búsqueda honrada vale mucho más que la presuntuosa seguridad de aquéllos que renuncian a la búsqueda porque creen ya saber - jsaber sin haber buscado por sí mismos!-. Nuestra preocupación debe ser meditar continuamente sobre el misterioso origen de la actividad artística. Empezando siempre desde el principio; observando una y otra vez, e intentando organizar y reagrupar las cosas de nuevo por nosotros mismos (1974, p. 2).

\footnotetext{
1 "Uno de los atributos naturales del hombre es la curiosidad. Se ha estado haciendo la pregunta «¿Por qué? » durante tanto tiempo que nunca se le ocurre pensar por qué lo hace, aunque a menudo corre el riesgo de desilusionarse" (Traducción de la autora).
} 
En el caso del intérprete, esta curiosidad es, prácticamente, una obligación determinada por su oficio, dado que, como se ha visto, no puede ejercer la completa libertad de un creador, sino someterse al pensamiento del compositor por medio de la obra de arte musical y de su correspondiente "paradigma estilístico" (Vela, 2017), de nuevo, movido por la curiosidad: "Richard Wagner me enseñó a leer partituras. Yo pronto quise saber cómo lo hacía, cómo funcionaba aquello, cómo surgían esos sonidos, esas increíbles mixturas sonoras" (Thielemann, 2013, p. 114), es decir, un punto de partida desde la misma obra musical:

\begin{abstract}
Puesto que en la música sólo tenemos lo escrito ante los ojos, no sólo el sabio, sino, en cualquier caso, todo intérprete realizador de lo escrito, si quiere hacer sonar la obra en forma válida, ante la obligación de llevar a cabo aquel proceso de pensamiento que conduce al descubrimiento de los detalles a los que la obra desde su forma y su especial composición, de la intención y de la forma en que nos va a estimular (Swarowsky, 1989, p. 12).
\end{abstract}

Muchas veces existen buenas razones para desatender las intenciones de un compositor. Pero no hay razón para no intentar averiguar cuáles eran sus intenciones o qué significaban para él las indicaciones que puso sobre el papel -incluso cuando no siempre haya sido consecuente, e, incluso, cuando en ocasiones, haya sido propenso a equivocarse, como todos- (Rosen, 2005, p. 16).

Por tanto, la enseñanza del intérprete requiere una formación musical integral que integre el contenido de los distintos paradigmas estilísticos, sus correspondientes "códigos simbólicos" (Vela, 2018) y "relaciones internas" (Ansermet, 2000) que los componen en tanto que galaxias musicales de elementos interconectados.

\title{
2. CONSIDERACIONES PREVIAS EN LA ENSEÑANZA DE LA DIRECCIÓN ORQUESTAL
}

Sin duda, la instrucción del director de orquesta debería una formación integral que comprendiese no sólo la interpretación de un instrumento sino también toda una serie de disciplinas teóricas que competen a la comprensión del discurso musical en su totalidad, es decir, a la ciencia de la composición. No obstante, desde algunos círculos de la actualidad se ha criticado el bajo nivel de formación del intérprete, con una notable caída de su instrucción, como recordaba Ros-Marbà:

Me he encontrado con algo que hubiera sido inimaginable en mi generación: era imposible que una persona pudiera ser director sin tener una formación teórica acabada. Escribir un cantus firmus, un contrapunto a cuatro voces, son cosas deficitarias hoy en día. En la mayoría de los casos no salgo de mi perplejidad ante la falta de soltura en estas disciplinas, sumamente importantes para el futuro director (Madigan, 2008, p. 112). 


\section{MARTA VELA GONZÁLEZ}

Sin embargo, desde el último tercio del siglo XIX, con el auge de los maestros de música profesionales, surgió una gran preocupación por la completa formación de los estudiantes en diversas disciplinas musicales, que acabó, poco a poco, con el dilettantismo sufrido durante décadas:

Lo máximo a que puedo aspirar es a recomendar ciertos procedimientos que rara vez serán erróneos, llamar la atención del estudiante sobre el hecho de que hay distinciones que hacer, y dar algunos ejemplos sobre cómo la evaluación de las progresiones armónicas les ayudará a reconocer sus propias deficiencias (Schoenberg, 1990, p. 19).

En aquella época, en los tiempos de Schoenberg, uno de los más insignes pedagogos del momento, un intérprete que se preciase de serlo recibía una educación sumamente completa, a pesar de que apenas existía un sistema reglado y un plan de estudios fijo, y, de este modo, la enseñanza sobre el contenido de la obra era irrenunciable para la cualquier instrumentista, director o compositor, como una artesanía del lenguaje de la música.

En efecto, en la educación de entonces, la enseñanza se orientaba a la comprensión del discurso -en el caso de la interpretación- y a la ordenación del discurso -en el caso de la composición- y, en cualquiera de los casos, era indispensable el conocimiento profundo de las relaciones internas de la obra para ejercer cualquier labor musical de carácter artístico en plenas condiciones:

Escuchando un concierto, a menudo me he encontrado a mí mismo, inesperadamente, en un "país extraño», sin saber cómo he ido a parar ahí; ha ocurrido una modulación que ha escapado a mi comprensión. Estoy seguro de que esto no me habría sucedido en tiempos pasados, cuando la educación de un intérprete no difería la de un compositor. Grandes directores como Nikisch, Mahler, Strauss eran conscientes de la alteración gradual de la textura que precede a una modulación y motiva un «cambio de escenario», la introducción de un contraste (Schoenberg, 1974, p. XVI).

No es casualidad que, hasta la primera mitad del siglo XX, buena parte de los compositores interpretaban y buena parte de los intérpretes componían, de hecho, la separación entre ambas parcelas estaba mucho más difusa que en la actualidad, dado que la enseñanza, en suma, era intercambiable entre compositores e intérpretes, de hecho, los ejemplos de compositores-intérpretes de esa época son innumerables, sobre todo, en el campo de dirección de orquesta: Mahler, Richard Strauss, Furtwängler, Hindemith, Stravinski, Webern, Bruno Walter, y un largo etcétera.

De este modo, los directores conocían los mecanismos de la composición musical y viceversa, una suerte de intercambio artístico sumamente fructífero: 


\section{APROXIMACIÓN METODOLÓGICA AL ESTUDIO DE LA DIRECCIÓN DE ORQUESTA} DESDE EL PUNTO DE VISTA DE LA OBRA MUSICAL

Componer es, para mí, poner en orden cierto número de estos sonidos según ciertas relaciones de intervalo. Tal ejercicio conduce a la búsqueda del centro hacia el que debe converger la serie de sonidos que utilizo en mi empresa. Me dispongo entonces, una vez establecido el centro, a encontrar una combinación que le sea apropiada, o bien, en el caso de tener ya una combinación todavía no ordenada, a determinar el centro hacia el que debe tender. El descubrimiento de este centro me sugiere la solución (Stravinski, 2006, pp. 43-44).

Schönberg hablaba siempre de la importancia de la inspiración para componer, pero inmediatamente hablaba en la necesidad de refrenar esa inspiración, que debería ser insertada en un sistema derivado de esa misma inspiración. Se sentía aquí actuar una síntesis maravillosa de sentimiento e intelectualidad (Swarowsky, 1989, p. 261).

De estas aseveraciones se desprende que cualquier músico, ya sea intérprete o compositor, debe saber cómo funciona la música. De este modo, todo músico debería conocer en profundidad el funcionamiento interno del arte del sonido, como se hacía en el pasado, incluso, como una íntima necesidad:

Tan pronto como [Riccardo Muti] decidió ser director de orquesta sintió la «acuciante necesidad» de estudiar composición y adquirir una información técnica sin la cual se habría sentido «falto de preparación». Fue una decisión valiente, pues en Italia los estudios de composición duran diez años y se componen de cuatro años de armonía, tres de contrapunto -lo cual comprende a todos los contrapuntistas flamencos, italianos y alemanes con sus respectivos estilos y contrapuntos a doce, trece o quince partes, dobles coros, etc. - y tres años de orquestación. El objetivo consiste en proporcionar un conocimiento de todas las formas compositivas formas desde las sonatas hasta las sinfonías, además de un profundo entendimiento de la instrumentación y de las posibilidades de cada instrumento (Matheopoulos, 2004, p. 285).

Por tanto, la labor de la enseñanza reside en ofrecer al alumno una formación integral que comprenda, antes que nada, las leyes fundamentales de la música, como recordaban insignes pianistas del siglo XX, Neuhaus: "he dicho ya que un profesor, tanto si enseña canto (asimilo la voz humana a un instrumento) o cualquier otro instrumento, debe ante todo enseñar, comentar y explicar la música" (1987: p. 164); o Deschausées: "todos estos elementos sensibles de la música [...] Forman parte del aprendizaje del músico. Pero, ¿por qué el aprendizaje del instrumentista -que poco a poco lo puede llegar a ser un virtuoso- no se completa con ese aprendizaje del músico que le permitiría convertirse en artista y en maestro? ¿No se produce aquí una grave amputación del ser? Cuando el trabajo ha sido orientado desde sus principios hacia la creación, no es necesario preguntar dónde están las carencias, las dominantes con las notas sensibles, ni qué significan las modulaciones" (Deschausées, 2009, p. 95). 


\section{MARTA VELA GONZÁLEZ}

En este punto, el análisis se muestra como una estrategia multidisciplinar -fundamental en el aprendizaje y práctica de la composición musical- cuya misión consiste en elucidar la labor del músico, es decir, facilitar su comprensión del lenguaje musical en pos de la verdadera expresión artística.

Por tanto, contemplamos la mencionada formación integral, desde la enseñanza del contenido de la obra musical a través de diversas herramientas analíticas como medio que ha de conducir al fin: la interpretación o la composición a su más alto nivel, en suma, la expresión del mensaje artístico, ante todo.

Berg era amigo de los análisis. En su juventud analizó escrupulosamente las obras de Schönberg: los Gurrelieder, Pelléas et Mélisande y la Primera Sinfonía de Cámara. Estos análisis acabaron siendo publicados, aunque durante mucho tiempo no fueron tan conocidos como merecían; el de la Sinfonía de Cámara, una pieza hoy tan difícil como antes, puede considerarse ejemplar; una publicación que reuniera todos estos análisis sería muy recomendable (Adorno, 2008, p. 361).

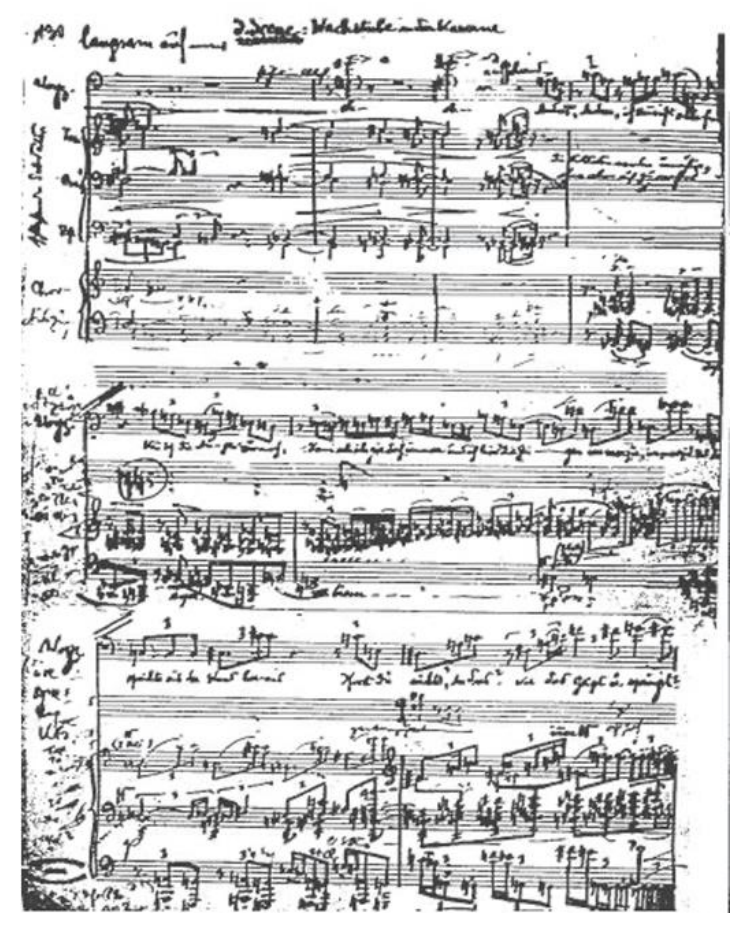

Fig.1: Berg, Wozzeck, manuscrito

(imagen procedente de imslp.org).

Lo que produce música verdadera es única y exclusivamente la capacidad inventiva, la imaginación es inspiración de una mente creadora -siempre y cuando un creador «tenga algo que expresar»-. No obstante, un estudiante nunca debería escribir notas áridas. En todo momento debe tratar de «expresar algo» (Schonberg, 1943, p. 4). 


\title{
APROXIMACIÓN METODOLÓGICA AL ESTUDIO DE LA DIRECCIÓN DE ORQUESTA
} DESDE EL PUNTO DE VISTA DE LA OBRA MUSICAL

En muchos casos, la expresión aludida por Schoenberg, la enseñanza del contenido de la obra, ha sido desplazada por un interés desmedido hacia la técnica instrumental. Buena muestra de esta situación es la cantidad de tratados teóricos que han proliferado, desde el final del siglo XIX, enfocados al conocimiento de la obra y el estilo, en cuanto al aprendizaje de compositores y directores, frente la inexistencia del mismo material, dirigido a intérpretes, más allá de los tradicionales manuales de técnica, repletos de ejercicios mecánicos y repetitivos que, en efecto, no expresan nada.

\section{METOdOLOGÍA DE ESTUdio FRENTE A LA OBRA MUSICAL}

El director de orquesta, a causa de su colosal labor frente a la orquesta, puede ser considerado como el intérprete por antonomasia y, por tanto, su metodología de aprendizaje de la obra -en la que incide de manera especial, como señalaba Previtali, el estudio de la composición- debería ser conocida por todos los instrumentistas: "el estudio de la dirección orquestal (género sinfónico y operístico) deberá realizarse simultáneamente con los últimos cursos de la composición" (2007, p. 2).

El privilegio del director, frente al resto de músicos -ya toquen solos o en conjuntoreside en el estudio de la obra previo al ensayo -como cualquier integrante de la orquesta- y en la escucha de la música desde fuera, dos estrategias que deberían ser practicadas, en la medida de lo posible, por el resto de los músicos.

Swarowsky, director y maestro de directores, expresó una idea parecida, en su tratado de dirección de orquesta, a través de tres condiciones diferentes, el estudio de la obra, su trasmisión a la orquesta y el respeto a la voluntad del compositor.

\begin{abstract}
Primera condición: conocimiento absoluto de la obra, no sólo del qué, sino ante todo del cómo y del por qué; dominio de la teoría de la música, conocimiento de los estilos de épocas anteriores, la cualidad de saber situarse en el mundo de los sentimientos y pensamientos de aquellas épocas, observación para reconocer la estructura de la obra, no sólo analíticamente, sino también en relación con los hechos individuales y temporales, capacidad de recapitulación y de llamada a la vida de la idea interna. Estas cualidades capacitarán al director a satisfacer la condición impuesta [...] Segunda condición del director: el dominio del aparato ejecutante y la facultad de transmisión de sus conocimientos de la obra a los ejecutantes [...] Como tercera condición se espera de un director de orquesta que tenga la fuerza necesaria para agradar la disposición formal y a los valores de expresión el grado de consecuencia y biodiversidad que se corresponda con la idea artística del autor, de forma que la obra suene como si hubiese sido interpretada por su autor («como si él mismo la hubiese compuesto», exige Mozart de un intérprete en una carta del 17 de enero de 1778) (Swarowsky, 1989, pp. 81-87).
\end{abstract}

Con el fin de adquirir una perspectiva musical más amplia, examinaremos a continuación las distintas facetas musicales del director de orquesta, así como su metodología de estudio en cuanto al contenido de la obra: 


\section{MARTA VELA GONZÁLEZ}

-El director como intérprete: implica un gran conocimiento de los medios sonoros y una interminable labor en su correcto equilibrio -dado que del director depende la encarnación de la partitura en sonido-, a través de un minucioso estudio de la obra musical y de distintas técnicas de organización del material musical:

La música es principalmente sonido. Primero viene el sonido articulado, el tono, el color, y, por fin, la estructura. También compositores contemporáneos como Wolfgang Rhim o Aribert Reimann, cuando se les pregunta, dicen que lo primero que oyen son colores, un timbre, una determinada constelación y sólo entonces empiezan a pensar en la forma. De ahí que yo nunca diría: «Rhythm is it!», sino siempre: «Sound is it!»² (Thielemann, 2013, p. 115).

Por lo demás, ante ese reposo, cuando mueves la música, lo que debería ser piano se transforma en un mezzopiano y un mezzopiano se transforma en un forte, porque el movimiento le aporta ese ímpetu añadido. Ésta es una de las razones por las que, cuando a veces necesito una determinada clase de sonido, en especial un piano, hago que la orquesta toque una nota durante largo tiempo, y siempre funciona, porque así ellos saben a qué atenerse, entienden lo que quiero y se sumergen en ese sonido. Si no lo hiciera así saldría un sonido diferente (Karajan en Mathepoulos, 2004, p. 158).

Por tanto, resulta evidente que el manejo de los medios sonoros de la orquesta requiere una larga formación musical y una dilatada experiencia que los directores adquieren tras años y años de estudio intelectual, por un lado, y de práctica de las técnicas de ensayo, por el otro: “¿Cómo logra destacar un director joven? Trabajando duro y bien, ensayando tanto como sea posible y acumulando un sólido conocimiento de las obras” (Thielemann, 2013, p. 108).

El director debe abarcar todo este repertorio y debe ser capaz de resucitarlo a su propia vida, al calor de su fuego sagrado. Por grande que sea su inteligencia, sin una vasta cultura su comprensión a día de quedar necesariamente ilimitada y fragmentaria. Es indispensable que el director poseía amplios conocimientos de la historia de la cultura y que tenga ideas muy claras acerca de los lazos que unen las producciones representativas de las diferentes épocas. Lo primero le ayudará a descubrir las condiciones históricas que contribuyeron a dar a las personalidades creado días sus facciones peculiares; lo segundo le enseñará a establecer una línea divisoria muy clara entre lo que son particularidades propias de la época y las que lo son de una personalidad determinada (Scherchen, 2005, p. 23).

-El director como docente: implica un continuado magisterio sobre sí mismo y sobre los demás integrantes de la orquesta en la elaboración de la obra musical y, por ende, en la forja

\footnotetext{
2 Thielemann se refiere al documental ;Rhythm is it! de 2004, dirigido por Thomas Grube y Enrique Sánchez Lansch, acerca de la Consagración de la primavera de Ígor Stravinski, interpretada por Simon Rattle y la Orquesta Filarmónica de Berlín.
} 
del sonido de las orquestas a lo largo del tiempo, como han reconocido Muti, Weingartner y Solti:

Sobre todo, [el director] deberá trabajar duramente para asegurarse de que los músicos aprenden a escucharse unos a otros mientras tocan, del mismo que se escuchan mutuamente los miembros de un cuarteto de cuerda. La orquesta es igual que un cuarteto de cuerda desde este punto de vista: ciento veinte personas que quieren sonar como cuatro. Si las diferentes secciones (cuerdas, maderas, mentales y percusión) se escuchan las otras, el conjunto que están será de mayor calidad y más preciso (Mathepoulos, 2004, p. 282).

Par un travail appliqué et infatigable, il avait transmis à l'orchestre sa façon d' interpreter les oeuvres musicales, à tel point qu'il atteignant une perfection dans le jeu d'ensemble jusqu'alors inconnue, tandis que la plus méticuleuse répartition des éléments orchestraux; cela élucidait la question de savoir si tel exécutant était meilleur que tel autre ou si telle particularité du chef était justifiqué. L'orchestre était comme un clavier que Bülow était seul à manier [A través de un trabajo diligente e incansable, él había transmitido a la orquesta su forma de interpretar las obras musicales hasta tal punto, que logró una perfección en el conjunto hasta entonces desconocida, a la vez de la distribución más meticulosa de los elementos orquestales; quedaba elucidada la cuestión de saber si tal o cual ejecutante era mejor que otro o si tal peculiaridad del líder estaba justificada. La orquesta era como un teclado en el que Bülow tenía a su disposición] (Weingartner, 1911, p. 11).

Así que, aparte del talento musical, [el director] necesita el don de un pediatra para el análisis mental. Y también debe ser un buen educador. Exactamente esa mente combinación lo que necesita. Ahora bien, es posible ser un buen director sin ser educador, porque hay directores que conforman orquestas y hay directores invitados. $\mathrm{Y}$, he hecho, hay directores nacidos para forjar orquestas y hay directores invitados; $\mathrm{y}$ yo soy, sin duda alguna, uno de los primeros: un director musical nato que trabaja constantemente en conformar y perfilar una orquesta. Me veo a mí mismo como un músico escultor y veo a los directores invitados como pintores que llegan, improvisan algo y después se van. Me gusta dejar mi sello en las orquestas, y por eso odio los compromisos cortos; de hecho, ya apenas los acepto (Matheopoulos, 2004, p. 312).

La faceta pedagógica, como pone de manifiesto el testimonio de Solti, supone una gran importancia en la labor cotidiana del director de orquesta, de ahí la circunstancia de que algunos directores se hayan mantenido durante décadas al frente del mismo grupo humano, dado que las grandes transformaciones en la música son lentas y silenciosas, y requieren, para su consolidación, de un determinado período de tiempo. 


\section{MARTA VELA GONZÁLEZ}

El propio Muti es un artista dotado de una gran energía que genera una intensa excitación y cuya capacidad para forjar orquestas, transformado el sonido de dos famosas formaciones musicales -o, mejor dicho, le ha permitido dejar en ella es la impronta de su sonido en un espacio de tiempo relativamente corto-. En el «sonido Muti», una de las características principales es la claridad es increíble agilidad que consigue arrancar de las cuerdas, fácilmente reconocible y que puede escucharse, en la actualidad, en la Philarmonia de Londres y en la Orquesta de Filadelfia y, desde 1982, también en la Orquesta del Maggio Musicale Fiorentino y en la Scala (Matheopoulos, 2004, p. 280).

Rattle permaneció dieciocho años con la Birmingahm Symphony Orchestra e hizo de ella una agrupación de talla mundial. La calidad de su ejecución orquestal mejoró inmensamente, al tiempo que su programación se convirtió en una de las atractivas innovadoras. También fue responsable de su traslado a su nueva sala de conciertos, el Birminghan Symphony Hall, toda una obra de arte. «Lo que este joven director ha conseguido en Birmingham debería servir de ejemplo (aunque probablemente no ocurra) de cómo dirigir una orquesta sinfónica y fomentar en el público el deseo de conocer repertorio amplio y audaz», escribió el Sunday Times (Matheopoulos, 2004, p. $357)$.

Por tanto, el director de orquesta se revela como un compendio de cualidades humanas y artísticas, regidas por una necesidad de adquirir una "cultura aristocrática" (Swarowsky, 1989), en su posición, tradicionalmente predominante, sobre el resto de los intérpretes, o una “sabiduría completa" (Stravinski, 2006).

\section{METOdOLOGÍA DE ESTUdIO FRENTE A LA OBRA MUSICAL}

Desde la perspectiva de la interpretación orquestal, por tanto, la obra musical se asemeja a un mosaico sonoro de pequeñas teselas que han de encajar progresivamente bajo la labor musical del director, de ahí la importancia del estudio de las relaciones internas de la música a través de parámetros.

Como han asegurado distintas personalidades de la dirección orquestal, el estudio intelectual de la obra previo al ensayo es insustituible y el conocimiento del funcionamiento técnico de la orquesta, depende también, en buena medida, del estudio del estilo y su evolución a lo largo del tiempo, con el objetivo final de la expresión artística, en las palabras de Weingartner, Walter y Ros Marbà:

Que le chef d'orchestre soit avant tout sincère vis à vis l'ouvrage qu'il fait exécuter, vis à vis de lui-même et vis à vis du public. Qu'il ne pense pas, dès qu'il ouvre une partition: quelle interprétation vais-je donner de cet ouvrage? Mais: qu'a voulu dire l'auteur? [Que el director sea, ante todo, sincero con el trabajo que ha hecho ejecutar, desde su punto de vista y desde el del público. Que no piense, en cuanto abra la partitura, ¿qué interpretación haré de esta obra? Sino: ¿qué quiso decir el autor?] (Weintgartner, 1911, p. 49). 


\section{APROXIMACIÓN METODOLÓGICA AL ESTUDIO DE LA DIRECCIÓN DE ORQUESTA} DESDE EL PUNTO DE VISTA DE LA OBRA MUSICAL

He hablado ya del respeto fanático [de Mahler] hacia la partitura; de todas formas, no le cegaba nunca hasta el punto de hacerle perder de vista su deber supremo, que era el de realizar las intenciones del compositor. En nuestros días se suelen aceptar bastante bien las modificaciones de este tipo, incluso si las opiniones divergen en cuanto a la forma en que tienen que hacerse. En el caso de Mahler, venían de la seguridad asombrosa con la que comprendía la significación interior de un pasaje, incluso cuando la instrumentación de la época siguiera haciendo algo oscura (Walter, 2002, p. 84).

Debemos ser sumamente conscientes de la topografía de la obra. Si te guías por las leyes naturales que están dentro del oído y de la conciencia de cada cual, de cada ser único e irrepetible, lo que tocas siempre sonará como si fuera la primera vez, y cada vez será también único e irrepetible. La experiencia será la de transcender lo material de que está hecha la obra (Madigan, 2008, p. 105).

La metáfora del mosaico respecto de la obra musical, que muchos directores utilizan, posee una doble significación en cuanto a la interpretación: por un lado, las teselas, los elementos que componen el todo - esto es, las llamadas relaciones internas - y, por el otro, la visión del conjunto de la imagen, dispuesta a materializarse en sonido a través de la unión de las teselas -es decir, desde el estudio intelectual de la obra y la integración de todos sus elementos-.

«Estudio muy, muy despacio, siguiendo una técnica como de mosaico»-contestó Solti. «En primer lugar, leía la partitura nota a nota. No hacía nada más que eso, sin formarme todavía una imagen mental de la obra, sólo leía. Tengo que ser muy metódico, porque soy vago y si sé que tengo que estudiarme doscientas páginas, ¡no comienzo nunca! Pero si tengo claro que tengo que estudiar veinte páginas al día durante diez días, entonces sí lo hago. Así me engaño un poco. Una vez que he visto toda la partitura, la leo de nuevo intento hacerme una primera representación mental del tempo, de las texturas y de las dinámicas. Lentamente, de todo ello surge la música. Pero necesito cuatro o cinco lecturas para formarme una cierta imagen ${ }^{3}$ de su perfil y de su sonido, acertada o no» (Matheopoulos, 2004, p. 326).

Por tanto, a partir de la imagen del mosaico como representación de la interpretación musical, el director debe ser capaz, desde su experiencia práctica y, por supuesto, desde su sabiduría completa, de combinar todos los parámetros musicales implicados en la obra musical, a partir del paradigma estilístico y de sus correspondientes códigos simbólicos, como han expresado directores de distintas generaciones, Furtwängler y Rattle:

\footnotetext{
${ }^{3}$ En efecto, no puede obviarse que la "imagen sonora" de la obra en la imaginación del director y, en realidad, de cualquier músico, está sometida a las condiciones acústicas del lugar de la interpretación: "No es nada fácil conseguir un buen forte o fortissimo en Bayreuth. Darlo todo menos un cinco por ciento; tal vez ésa sea la fórmula. Y así se van juntando despacio y a base de práctica interpretativa las partes del mosaico" (Thielemann, 2013, p. 75).
} 


\section{MARTA VELA GONZÁLEZ}

En una sinfonía de Beethoven en cambio, el primer compás apunta ya al quinto, octavo, vigésimo, trigésimo, y contesta a otros pases hasta el final, y así a través de toda la obra. El compás aislado es sencillo, pero la invitación en el interior de los compases, en el interior de los temas, los cientos de correspondencias y variaciones, de gradaciones de la intensidad dinámica, que son el resultado del más genial escuchar en la lejanía, acaban produciendo una masa de complicaciones que, comprendida cabalmente, sobrepasa todo cuanto el ja₹₹ puede ofrecer (Furtwängler, 2012, pp. 197-198).

Esta apropiación paulatina de una verdadera comprensión musical es el desafío que afronta todo director. La técnica es fácil, aunque lógicamente se requiere tiempo para aprenderla. Pero una vez nos hemos enfrentado al auténtico problema musical, la cuestión se complica. Yo, por ejemplo, a medida que me voy haciendo mayor, me siento menos capaz de dirigir las sinfonías de Beethoven, algo para lo que antes era mucho más atrevido. Pero ahora que voy siendo más consciente de todos los niveles que las integran, de su inconmensurable profundidad, me sobrecoge la enormidad que tengo que asimilar (Matheopoulos, 2004, p. 341).

Con todo, el punto de partida de la reconstrucción del mosaico debe ser el estudio analítico de la obra, en un largo proceso donde se intercalan las distintas perspectivas de la interpretación:

1) No preocuparse nunca de crear una interpretación.

2) La interpretación es el resultado de un estudio profundo de la obra musical y puede resumirse en varias fases:

a) Estudio de la obra bajo el punto de vista técnico (melodía, armonía, contrapunto, posibilidades instrumentales y vocales).

b) Estudio del concepto general de la ejecución sobre la base de la propia intuición musical.

c) Estudio global de la obra con el objeto de localizar los posibles defectos de construcción que se puedan corregir mediante la ejecución.

d) Estudio de las posibles fuentes de inspiración, ya sea bajo el punto de vista musical histórico (derivaciones) o desde el punto de vista literario.

e) Selección de la característica que se considere más apropiada según la interpretación intuitiva (b) y la que se habrá fijado mediante los procedimientos (c) y (d) (Previtali, 2007, p. 22). 
De este modo, como se puede apreciar, no son tantas las divergencias entre el trabajo del director de orquesta y el trabajo individual de cualquier músico, especialmente, aquí yace la mayor paradoja, del pianista, que contempla ante el atril, en muchas ocasiones, un discurso musical de gran fluidez, pero de suma individualidad en la interpretación.

\title{
5. ANALOGÍA ENTRE EL PIANISTA Y EL DIRECTOR DE ORQUESTA
}

Tradicionalmente, el piano ha sido considerado como el sustituto natural de la orquesta, en efecto, se trata de un medio accesible, doméstico y barato, de ahí que, en el pasado, todas las obras orquestales se redujesen -con un evidente interés comercial-, en una partitura pianística de dos o cuatro manos para ser ejecutada en el hogar burgués del siglo XIX.

Así lo han refrendado diversos pianistas que habían sido educados desde 1880: "me gusta repetir a mis alumnos lo que Anton Rubinstein decía del piano: «¿Creéis que es un solo instrumento? Es un centenar de instrumentos»” (Neuhaus, 1987, p. 64); "su extensión abarca más de seis octavas, vale decir, supera a la mayor de las orquestas, pudiendo, sin embargo, ser ejecutado este enorme material sonoro por los diez dedos de una sola persona, mientras la orquesta requiere el trabajo de cien ejecutantes" (Casella, 1936, p. 67); “en el piano, más que en otro instrumento de cuerdas alguno, tenemos un muy estimable sustituto de la orquesta completa, por cuanto lo mismo es capaz de matizar a voluntad la fuerza, la intensidad del sonido, quiere realizar un juego polifónico considerable" (Riemann, 2005, p. 7).

Por medio de un prolongado estudio del sonido del teclado, el pianista puede producir, gracias a la amplitud de registro de su instrumento, tal vez, sólo igualada por la del órgano, un amplio espectro de timbres, semejante a los de los instrumentos de la orquesta, así como reproducir la potencia de una orquesta entera, a causa de la condición percusiva del piano y la función de los pedales:

\begin{abstract}
Ahora bien, el piano brinda posibilidades insospechadas de sonidos, de colores, de vibraciones. Todo es posible para quien sabe tratar un teclado y utilizar sus recursos: sonido de campanas, batir de timbales, timbres de flauta, de arpa, de violonchelo... El piano-macillo se transforma entonces en un «piano-orquesta». Pero este logro exige horas, años de búsqueda y de receptividad, un verdadero trabajo de escuchar, una aproximación sonora cada vez más agudizada debería enseñarse con el mismo merecimiento que la técnica instrumental; puesto que cualquier trabajo que se realice un instrumentista al que hará su realización formal, se quiera o no, en la emisión de sonidos (Deschausées, 2009, p. 104).
\end{abstract}

Con él, una sola persona puede tener dominio de todo un completo y sobrepasar a cualquier otro instrumento al producir más fuerte y más suave de los sonidos en un sólo registro; las trompetas pueden gritar, pero no murmurar, las flautas al revés. El piano 


\section{MARTA VELA GONZÁLEZ}

puede hacer ambas cosas. Admite los más altos y los más bajos sonidos que se puedan obtener. El piano debería ser estimado (Busoni, 2004, p. 157).

Si en ocasiones se analizan las dificultades interpretativas del piano a causa de la condición percusiva del instrumento, también se debería hablar de sus grandes virtudes, fundamentalmente, de su capacidad ilimitada de reproducción de timbres -que ningún otro instrumento posee-, y de su capacidad de reproducción polifónica y, por ende, orquestal:

El piano es un lugar de transformación. Cuando el pianista así lo desea, el piano permite sugerir la voz humana en el canto, el timbre de otros instrumentos, la orquesta, el arco iris, las esferas. Esa capacidad de transformación, esa alquimia, es nuestro mayor privilegio (Brendel, 2013, p. 98).

También en el piano tenemos una gran abundancia de timbres. Si bien se discute aún sobre la antigua cuestión de si hay un timbre variable Con independencia de la dinámica de la nota aislada en el piano, es evidente que gracias a los pedales existe un inmenso margen de libertad con los timbres. ¿Cómo si no es posible que con uno y el mismo instrumento dos pianistas sean capaces de crear efectos sonoros tan diferentes, como si tocasen en dos instrumentos distintos? La pulsación, la articulación y el pedal son tres variables que se complementan y que están aquí a nuestra disposición. Ha de añadirse la ejecución dinámica de acordes en el piano mediante la cual, para uno y el mismo corte, pueden generarse timbres completamente diferentes, según cual sea la voz que lo mine el espectro sonoro (Mantel, 2010, p. 88).

Estas premisas explican, como ha afirmado Casella, la profusión y calidad del repertorio pianístico, no sólo como instrumento solista, sino como acompañante de todos los demás en sustitución de la orquesta, música de cámara, correpetición vocal y transcripción de todo tipo de música orquestal:

Podemos afirmar, indudablemente, que el piano, después de Liszt, resulta ser el medio fónico-expresivo que reúne en si la mayor suma de recursos y posibilidades que nunca jamás haya un ofrecido un instrumento musical. Tal superioridad sobre los demás instrumentos está abundantemente demostrada por la vasta literatura pianística Si se compara con la de otros instrumentos, por su superioridad en cantidad y calidad, lo que nos demuestra el grado de confianza que han sabido depositarle todos los compositores, desde Clementi hasta el presente (Casella, 1936, p. 66).

En este punto, se debería admitir la analogía entre el piano y la orquesta, en tanto que medios de expresión musical, y del pianista y el director de orquesta, en tanto que intermediarios de los creadores que han fiado su labor compositiva a estos soportes instrumentales. 


\title{
APROXIMACIÓN METODOLÓGICA AL ESTUDIO DE LA DIRECCIÓN DE ORQUESTA
}

DESDE EL PUNTO DE VISTA DE LA OBRA MUSICAL

El pianista, al igual que el director, puede tener dos roles distintos en cuanto a la función musical desempeñada: el pianista-intérprete, que debe saber moldear la materia musical a partir de su conocimiento teórico-práctico, y el pianista-docente, que debe, de la misma manera, forjar su propio sonido pianístico y, a su vez, puede influir directamente en sonido el de otros, gracias a la gran cantidad de labores musical que puede asumir un pianista en el amplio espectro docente de nuestros días: repertorista, correpetidor, profesor de música de cámara o piano complementario... y un largo etcétera.

\begin{abstract}
Dejando a un lado los movimientos físicos, el sonido queda determinado en su mayor parte por el equilibrio de las notas. Este equilibrio tiene que satisfacer las exigencias musicales del carácter, de la atmósfera, de la exposición anímica. Simultáneamente, la conciencia de los contrapuntos, es decir, la polifonía, influirá en el sonido. El intérprete debería aprender, sobre todo, de las obras orquestales, vocales y de música de cámara. El equilibrio de un buen sonido orquestal deberías ser nuestro modelo (Brendel, 2013, pp. 124-125).
\end{abstract}

Frecuentemente digo a mis alumnos, tanto a los menores, los adultos: «el pianista y su piano es a la vez: primero, el director de orquesta, es decir, la cabeza, el corazón y el oído; segundo, los miembros de la orquesta, es decir, las dos manos con sus diez dedos y los pies sobre los pedales, tercero, el instrumento, es decir, un sólo y único piano o, para retomar la palabra de Rubinstein, los cien instrumentos, tantos como hay una orquesta sinfónica» (Neuhaus, 1987, p. 72).

$\mathrm{Y}$, sin embargo, la analogía entre la formación del director y la del pianista no es un concepto nuevo: muchos músicos en el pasado han sugerido un enfoque metodológico similar en ambos casos, basado en dos premisas ya reseñadas, a saber, la necesidad de una formación integral a partir de una idea de cosmovisión musical, por un lado, y un proceso de estudio a partir de una imagen mental previa elaborada desde el conocimiento intelectual del intérprete, por otro.

Sugiero al alumno estudiar una obra para piano como un director de orquesta estudia una partitura, es decir, no solamente en su conjunto (esto ha de ser lo primero, en caso contrario, la imagen carecería de unidad), sino en el detalle, descomponiendo los detalles de las obras para estudiar su estructura armónica y polifónica, es decir, examinando lo esencial, su línea musical, y lo «secundario», el acompañamiento (Neuhaus, 1987, p. 33).

Remontarse a la vida del texto mediante un profundo conocimiento de la escritura musical. En cierta manera, hacer el mismo trabajo que un director de orquesta: cuando éste se presenta delante de los músicos, sabe lo que quiere transmitirles; él ha "oído" la partitura en su ejecución final y sabe el trabajo que deben desarrollar. Para un intérprete es lo mismo: su trabajo dará lugar a una auténtica creación si has sabido penetrar antes en el corazón de la obra la música es siempre la que debe orientar el trabajo técnico (Deschausées, 2009, p. 46). 


\section{MARTA VELA GONZÁLEZ}

De quienes más podemos aprender los pianistas es de los directores y de los cantantes. Mientras que el cantante nos recuerda la necesidad tanto de cantar como de hablar, el director nos ofrece la orquesta como modelo de equilibrio, color y ritmo (la imagen del pianista como una orquesta de diez dedos procede de Hans von Bülow). Es posible dirigir nuestras modificaciones del tempo mientras la pieza no exija alguna improvisación. ¡En nuestra mente somos nuestro propio director! La orquesta puede ser un correctivo para la ligereza rítmica de algunos intérpretes de piano excesivamente solistas (Brendel, 2013, p. 42).

Hasta tal punto llega la analogía que, incluso, los errores interpretativos de pianistas y directores pueden ser los mismos, como, por ejemplo, en la falta de escucha:

No hay quizás otros músicos -con excepción de los directores de orquesta- que sean tan poco conscientes como los pianistas de cómo suenan realmente sus interpretaciones. Esto se debe a que una gran parte del sentimiento que pertenece, con razón, al sonido de la música se encarna, para los pianistas y para los directores, con el esfuerzo físico, en el gesto (Rosen, 2014, p. 51).

El hecho de no saber escucharse a sí mismos, no es solamente exclusivo defecto de los alumnos. Hay también ejecutantes y directores de orquesta que, evidentemente, no perciben lo que hacen, pero, sí sienten en su fantasía, la música como quisieran que fuera, y que, por desgracia no es (Casella, 1936, p. 100).

De esta forma, el proceso de estudio de pianista y director debería ser dirigido por la imagen mental previa al sonido, enriquecido por otros elementos derivados de un conocimiento intelectual de la obra musical, es decir, la escucha interna, la imaginación, el tempo y, en suma, los objetivos interpretativos al más alto nivel, basados en la transmisión de la idea del compositor correspondiente:

Todo este trabajo mental es similar a la preparación que realiza un director antes del primer ensayo con orquesta. Ahora podemos abordar el primer ensayo -la primera sesión de prácticas- con los fundamentos esenciales en su sitio: un esbozo de una concepción; un conocimiento del contexto, de qué que es lo que ocurre en la música y por qué; y, lo más importante, la habilidad de escuchar la música en todas sus dimensiones, incluso, los problemas técnicos pormenorizados se habrán anticipado y resuelto al menos parcialmente (Rink, 2006, pp. 163-164).

Esta particularidad precisamente, que hace que el piano, lo mismo que aquellos instrumentos antiguos, más que reproducir los sonidos reales, los imita, los apunta y, por tanto, reclama el más alto grado la colaboración de la imaginación sonora del oyente, como entendemos que radica el principal y más singular mérito de nuestro instrumento (Riemann, 2005, pp. 66). 
Leimer fue uno de los primeros pianistas que anticipó la idea de la imagen previa al sonido en el estudio de la obra para piano. Desarrolló entonces una exitosa metodología de estudio -ejemplificada en el más famoso de sus alumnos, el genial pianista Gieseking-, calificada como moderna ejecución pianística, en contraste con todas las tendencias de tipo dilettante del siglo XIX, cuya primera premisa consistía en memorizar la obra desde un proceso previo de reflexión, al igual que el director de orquesta:

Condición indispensable para ese adiestramiento del oído es el exacto conocimiento de la imagen escrita de la obra a estudiarse (...) Ahora bien, para lograr esto en forma rápida es necesario también un entrenamiento especial de la memoria. Para ello utilizo ampliamente la reflexión (2007, p. 14).

Desde entonces han sido muchos los músicos de todo tipo los que consideran necesario un estudio desde la imagen mental como paso ineludible en el proceso de interpretación de la obra musical, no ya sólo en el ámbito de la dirección orquestal, sino también en cualquier instrumento y en el canto:

El bajo-barítono Thomas Quasthoff comenta en su autobiografía: «Siempre que trabajo un nuevo lied, medito primero acerca de qué timbres puedo utilizar para poner de manifiesto de la mejor manera posible la composición». Destaquemos que Quasthoff, más allá de los timbres vocales normales (es decir, de los informantes), piensa en otras colaboraciones que sostengan el carácter y el ánimo de la música (Mantel, 2010, p. 89).

Nos encontramos en un proceso que puede describirse como un ciclo: el resultado sonoro, la propia interpretación escuchada y experimentada, es siempre una reacción a la idea interpretativa, a la concepción sonora. No es, como a menudos se cree, el resultado de una concepción absoluta y traducida posteriormente con la técnica disponible en ese momento ( $y$, eventualmente, limitada por nuestras deficiencias) (Mantel, 2010, p. 29).

Por tanto, un labor tan colosal y minuciosa como la comprensión de la obra musical compuesta por elementos teóricos y prácticos-, precisa de una honda reflexión en el proceso de estudio, desde distintas técnicas analíticas, y de una profunda cultura aristocrática que sólo se consolida con el paso del tiempo.

Ésta ha sido la preocupación de distintos artistas desde la segunda mitad del siglo XX, a causa del frenético ritmo de la vida moderna que nada tiene que ver con la interpretación musical ni su lento aprendizaje, todo lo contrario, en el sentir de Guilini y Karajan:

Guilini creía que el artista debía «defenderse del trabajo excesivo», y consideraba que uno de los mayores problemas de nuestra época es que no nos concedemos el tiempo para pensar. La palabra acción expresaba esta idea, y volar era relativamente fácil y 


\section{MARTA VELA GONZÁLEZ}

cómodo, pero demasiado rápido para su gusto. «Cuando era joven, necesitaba tiempo para estudiar. Ahora necesito tiempo para pensar» (Matheopoulos, 2004, p. 118).

En ese sentido con nuestra profesión es una de las pocas de nuestro tiempo que va a contracorriente. Hasta hace poco, sobre todas las civilizaciones milenarias como China, el hombre quería envejecer, porque es entonces cuando se le consideraba un sabio y todos debían guardar silencio si tomaba la palabra. Pero en estos tiempos las cosas son al revés, no sabemos todavía si para bien o para mal pero así lo dicta las exigencias de nuestro mundo moderno. Sin embargo, hay ciertas cosas, como nuestra profesión, que son una excepción a la regla, porque no pueden aprenderse con rapidez (Matheopoulos, 2004, p. 168).

Precisamente, ese trabajo excesivo aludido por Guilini es el generado por la honda reflexión de la obra, surgido de la recreación individual y solitaria de la imagen sonora de la música.

\section{LA IMAGEN SONORA DE LA MÚSICA}

La metodología de estudio del director, por tanto, está basada en un minucioso trabajo analítico previo al momento en que la obra se convierte en sonido. De esta manera, cuando el director sube al podio el primer día de ensayo, la mayoría del trabajo interpretativo ya está perfectamente estructurado en su cabeza, así como la secuencia de todas las estrategias musicales que utilizará durante los ensayos para dar vida a la partitura. De hecho, sería deseable que todo director tuviera una formación pianística lo suficientemente profunda como para poder utilizar en el instrumento en el estudio de la obra orquestal.

Berlioz, uno de los primeros directores de orquesta profesionales de la historia, junto a Mendelsshon y Wagner, enunciaba, durante la primera mitad del siglo XIX, todas las labores a que debía enfrentarse el director en el transcurso de un ensayo:

El director de orquesta debe ver y oír, debe ser ágil y riguroso, conocer la composición, la naturaleza y la extensión de los instrumentos, saber leer la partitura y poseer, además del talento especial del que intentamos explicar las cualidades constitutivas, otros dones casi indefinibles, sin los que un vínculo invisible no puede establecerse entre él y aquéllos a quienes dirige... (Llort Llopart, 2005, p. 157).

En este lento proceso de aprehensión de las relaciones internas de la música -en que el análisis juega un papel fundamental-, el estudio del director, previo al ensayo, se orienta, en primer lugar, hacia la formación de una imagen ideal de la obra, de carácter sonoro y mental, que trata de recrear, al detalle, todos los elementos de la futura interpretación: "una partitura no puede interpretarse. Primero hay que leerla correctamente y después describirla de diversas maneras. De este modo puede desarrollarse la idea de una configuración. Esta idea es lo que puede interpretarse" (Mantel, 2010, p. 36). 


\section{APROXIMACIÓN METODOLÓGICA AL ESTUDIO DE LA DIRECCIÓN DE ORQUESTA}

DESDE EL PUNTO DE VISTA DE LA OBRA MUSICAL

Esta imagen sonora, pero previa al sonido mismo, se corresponde con la imagen estética de Neuhaus o la imagen gráfico-musical de Leimer -resulta curioso que ambos hablen de un objeto visual, la imagen, que posteriormente se transforma en sonido-, sobre la que no sólo se debe trabajar desde el principio en la preparación de una obra, sino, también, desde el inicio de la educación de todo músico, con un minucioso estudio de la partitura:

Para la comodidad de la exposición me resuelvo a alejar la duda que me obsesiona a propósito de la fórmula «trabajo sobre la imagen estética». Tomémosla por válida y pongámonos de acuerdo sobre el enunciado siguiente: el trabajo sobre la imagen estética comienza desde los primeros pasos en el estudio de la música y del instrumento (Neuhaus, 1987, p. 23).

La fijación exacta de la imagen gráfico-musical mediante el trabajo mental para el logro de la técnica, es el primer problema a resolver. La manera más rápida y acabada de alcanzar esto la proporciona la concentración intensa de todas las facultades mentales. Es, pues, un trabajo cansador (Leimer, 1951, p. 14).

En el caso de la dirección orquestal, esta imagen estética y sonora, que comprende todos los elementos comprendidos en el paradigma estilístico desde una perspectiva intelectual del estudio de la música, es un paso insustituible en la transformación de la partitura en sonido, a causa de la gran cantidad de labores musicales y humanas que recaen sobre la figura del director, en opinión de Abbado:

En primer lugar, conocer realmente bien la obra. Nunca se la llega a conocer bastante. Siempre hay algo más que aprender. El director de saber todo lo que se conoce sobre el compositor, y esto también debería incluir toda su obra de cámara y vocal, para tener una mejor idea de su estilo. También ha de saber tocar un instrumento, haber estudiado composición, tener un buen sentido del ritmo y buen oído; psicología para tratar con la gente; tenacidad. Es importante amar lo que se está haciendo, tener pasión por la música; de otro modo, el trabajo se volvería rutinario y sería el fin, la muerte. También debería entender y respetar a otros músicos y cantantes cuyas personalidades puedan ser diferentes de la suya. Esto se hace especialmente necesario cuando se trabaja en una ópera, en la que participa tanta gente, o con solistas, porque es imposible que dos o más personas tengo exactamente la misma idea de una hora determinada, y los grandes solistas son grandes personalidades. De modo que un director debe comprendernos y comunicarse con ellos a un nivel muy profundo (Matheopoulos, 2004, p. 56).

La formación mental de esta imagen de la obra requiere un gran esfuerzo de imaginación sonora, así como un largo período de reflexión sobre la misma, que marca la génesis de la futura interpretación orquestal: 


\section{MARTA VELA GONZÁLEZ}

El director debe tener una audición interna de la obra tan perfecta como la tuvo su autor. El artista creador levanta su obra con materiales espirituales; él descubre nuevas coloraciones del sonido, tiene una intuición nueva de la materia; los sonidos reciben la impronta de su personalidad (Scherchen, 2005, p. 3).

Me llevó semanas meterme en mi oído esa estructura melódico-armónica [la del Concierto para violoncello de Frank Martin], es decir, ser capaz de oírla en mi cabeza antes de oírla tocada por los instrumentos; y es así como entiendo el trabajo del director de orquesta; debe tener la música en su interior si quiere controlar su ejecución (Ansermet, 2000, p. 145).

Por tanto, podríamos resumir el proceso de interpretación desde el punto de vista del director de orquesta en dos fases separadas en el tiempo: la formación de la imagen a través de un estudio intelectual y su encarnación en sonido desde el primer ensayo y, posteriormente, su ejecución pública, como explicaba Scherchen:

En mayor medida que otro artista cualquiera, el director de orquesta ha de poseer un dominio soberano en la representación mental de la partitura, ha de ser capaz de recrear en su fantasía la imagen sonora ideal de la obra. Sólo cuando haya logrado eso, cuando la obra haya adquirido suma perfección esta recreación imaginativa, puede atreverse el director a darle forma plástica por medio de la orquesta. Distinguimos dos frases en este proceso: una fase preliminar, de preparación, durante la cual el director disculpe en su fantasía la obra que desea producir, realizándola in mente con el más elevado grado de perfección que es capaz de imaginar; y la segunda fase, la de la realización de esa representación ideal, la dirección de la obra, la solución práctica de los problemas de interpretación instrumentales (2005, pp. 1-2).

De esta forma, el director sintetiza en esta imagen mental no sólo su conocimiento de la obra en tanto que código escrito, sino que recrea en su imaginación, a su vez, una multiplicidad de elementos musicales, en su mayoría, derivados de un profundo estudio intelectual de la obra, en tanto que código artístico.

Durante el proceso de la formación de la imagen mental director se ponen en juego otros factores derivados de la experiencia musical: memoria, fantasía, audición interna, tempo, etc., que han de ser sometidos, por tanto, al estilo correspondiente y, por supuesto, también a la personalidad del intérprete, en tanto que co-creador de la obra musical, con la colaboración de la orquesta, conforme el criterio de Böhm:

Todo esto de dirigir a una orquesta, en torno a lo cual tanta gente quiere construir una especie de mística, no es más que un trabajo. Toda la partitura la estudio muy, muy detenidamente -éste es el primer requisito: conocer las partituras, no creer que las conoces, sino conocerlas de verdad-y tener en la cabeza una idea clara sobre el tempo que voy a llevar. Y así se forma una imagen ideal de la partitura en la mente. Intento 


\section{APROXIMACIÓN METODOLÓGICA AL ESTUDIO DE LA DIRECCIÓN DE ORQUESTA} DESDE EL PUNTO DE VISTA DE LA OBRA MUSICAL

alcanzar este ideal que, por supuesto, nunca lo alcanzo. Si consigo, digamos, un $90 \%$ de esta imagen ideal, me doy por contento, pues el resultado se ajustará más o menos a mi concepción. ¡Pero eso depende de tantas cosas! (Matheopoulos, 2004, p. 94).

Qu'il étudie la partition à fond, de façon qu'elle ne soit plus pour lui pendant l'exécution qu'un aide pour sa mémoire et non une entrave pour ses pensées. L'étude de l'oeuvrage lui a-t-elle donné une image, qu'il la donne dans son unité et non morcelée [Que estudie la partitura a fondo, de manera que durante la ejecución no sea sino una ayuda a la memoria y no obstáculos para sus pensamientos. El estudio de la obra le ha dado una imagen que él transmite en su unidad y no fragmentada] (Weingartner, 1911, p. 49).

Por tanto, en un sentido intelectual, la imagen de la obra se ha de moldear constantemente a través de un cuidadoso análisis que trate de hallar las relaciones internas de la música y su jerarquía a través de parámetros, a saber, estructura, forma, secciones, frases, desarrollo armónico..., tal cual lo explicaba Leimer a comienzos del siglo XX:

Es una experiencia pedagógica importante el limitarse, al comenzar el estudio de una composición, únicamente a uno de los problemas que se presentan, pues, de otra manera, las diferentes tareas se piensan demasiado superficialmente y no se realizan con todo el detalle que requieren. Cuando se trata de alumnos muy adelantados, la consideración simultánea de varios problemas es ciertamente posible y acaso también conveniente. El estudio se hace progresivamente en la siguiente forma:

1. Se ocupa uno exclusivamente de grabar en la mente la imagen gráfico-musical;

2. Se compenetra uno con máxima exactitud de los valores de las notas;

3. Se aplica a lo ya dominado los adecuados géneros de toque;

4. Se pasa entonces al estudio de la interpretación, cuando aquellos problemas preliminares han sido resueltos en forma cabal.

Una ejecución pianística de primera clase es sólo imaginable y posible cuando esas tareas se han realizado con máximo cuidado y absoluta exactitud. Esta solución progresiva de los problemas, que debe buscarse con la más extrema concentración, es no sólo el procedimiento que lleva a la meta con mucho mayor prontitud, sino que proporciona también la mejor y más rápida manera de penetrar en las intenciones del compositor, y de realizarlas (1951, p. 7).

Veamos, pues, un ejemplo de la metodología de estudio de Leimer, sobre un diagrama explicado paso por paso, procedente del primer movimiento de la Sonata para piano Op. $2 n^{\circ}$ 1 de Beethoven:

Tomemos como ejemplo la primera Sonata en Fa menor. Para su memorización podrá ser de ayuda en la siguiente meditación: la sonata empieza con el arpegio de fa menor desde do hasta la bemol. Siguen luego las notas de un grupeto sobre fa en el segundo compás. Viene después el acorde (arpegio) de séptima de dominante (sol hasta si bemol) con las 


\section{MARTA VELA GONZÁLEZ}

notas subsiguientes del grupeto sobre el sol, luego la repetición de los compases segundo y cuarto, y, enseguida de éstos, el acorde de fa menor en posición cuarta y sexta, al que siguen corcheas en escala descendente hasta mi. En la mano izquierda alternan acordes de tónica de fa menor con otros de séptima dominante; son fáciles de recordar. Estos ocho primeros compases del tema principal son fáciles de retener a la lectura mediante la reflexión, y pueden y deben tocarse de memoria y luego estudiarse. Una vez que uno se ha acostumbrado a retener por la meditación esos tales pasajes, se desarrollará paulatinamente la facultad de oír también esos fragmentos con el oído interno a la simple lectura. Con ello se pone en buenas condiciones de captar y comprender de inmediato las ideas contenidas en la composición, de manera que el trabajo, mecánico en un principio, se convierte luego en tarea seguramente espiritual. El alumno deberá tratar de alcanzar poco a poco esa alta meta, (Leimer, 2007, p. 28).

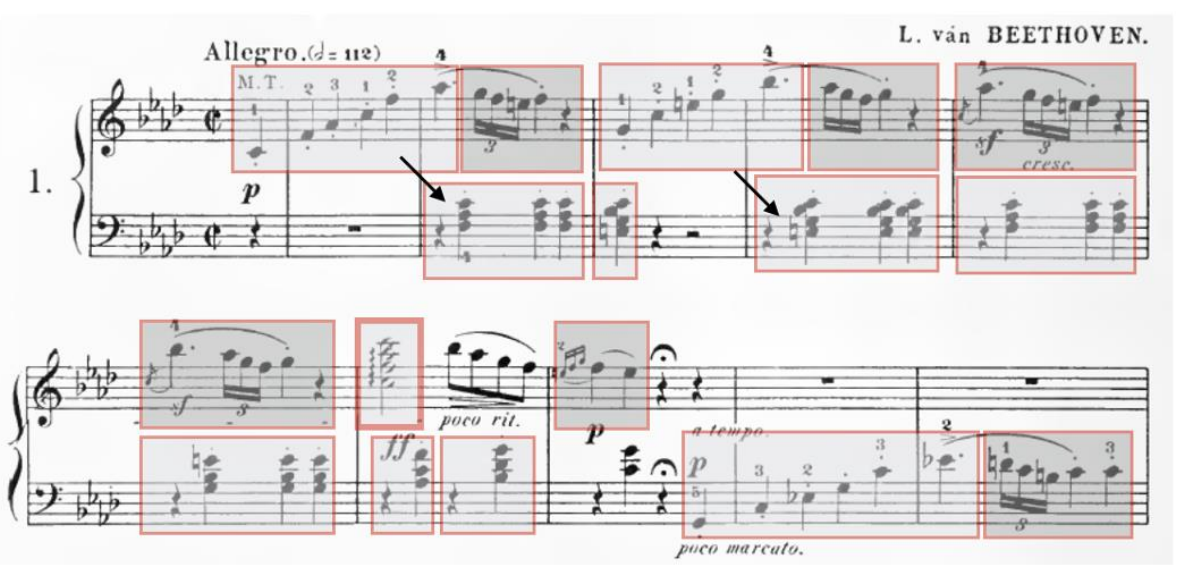

Fig. 2: Beethoven, Sonata en Fa menor Op. $2 n^{\circ} 1$, I

(imagen procedente de imslp.org).

De igual modo, el período de reflexión en que el director forja su imagen mental de una obra es, en gran medida, un proceso analítico de distintos niveles consciencia progresiva, como opinaba Bernstein:

Pero si me enfrento a una nueva obra por primera vez, suelo leer la de principio a fin como si fuera una novela de misterio, una historia de detectives, la estudio detenidamente para saber qué es lo que va a pasar. Madre mía, el suspense por lo que vaya a pasar después, por lo que va a pasar ahora. ¿Cómo terminarán el segundo y el tercer movimiento, cómo va a transcurrir todo? [...] Luego, cuando ya tengo esa forma básica en la cabeza, comienzo de nuevo, analizándola ahora con detalle, no memorizando las notas compás por compás o frase por frase; simplemente la analizo. La memorización viene por sí sola y, cuanto mejor sea la partitura, más fácil es memorizarla [...] Después de analizar la estructura y la relación entre cada uno de los componentes, puedes centrarte en los detalles. El trabajo es interminable. Y cuanto mejor sea la obra, más interminable será su estudio (Matheopoulos, 2004, pp. 32-41). 
Entre tanto, el proceso de estudio se desenvuelve junto con otros elementos de carácter intelectual que resultan imprescindibles en toda interpretación musical, como, por ejemplo, la memoria:

Hay que ir de lo general a lo particular haciéndose poco a poco con la obra hasta que se tiene asimilada en la conciencia y, para mí, al menos, para esto es imprescindible memorizar, si no todas, al menos las grandes obras. Es una necesidad visceral. Si no lo hago, me auto-traiciono. La obra no es mía, aunque para que lo sea necesito un largo período de reflexión (Madigan, 2008, p. 138).

En suma, cuanto más elaborada, técnica y sonoramente, sea la imagen creada por el director en su proceso de estudio, más completa y artística será la versión encarnada de la música y, evidentemente, este trabajo previo en profundidad depende, casi íntegramente, del bagaje cultural del director y de su capacidad analítica para obtener conocimiento de los "códigos simbólicos" y del "paradigma estilístico" correspondiente, de nuevo, en palabras de Bernstein:

Así, cuando me puse con la Novena de Beethoven por enésima vez hace dos semanas, dije que le dedicaría una hora después de cenar, para repasarla un poco y refrescar mi memoria antes de irme a la cama a medianoche. Ja! Después de media hora, todavía estaba en la segunda página y todavía continuaba con la misma a las dos de la mañana y, de ningún modo, cerca del finale, como verá. Estaba en algún punto del adagio, perdido en las estrellas, porque estaba encontrando muchas cosas nuevas. Era como si nunca la hubiera visto antes (Matheopoulos, 2004, p. 40). 


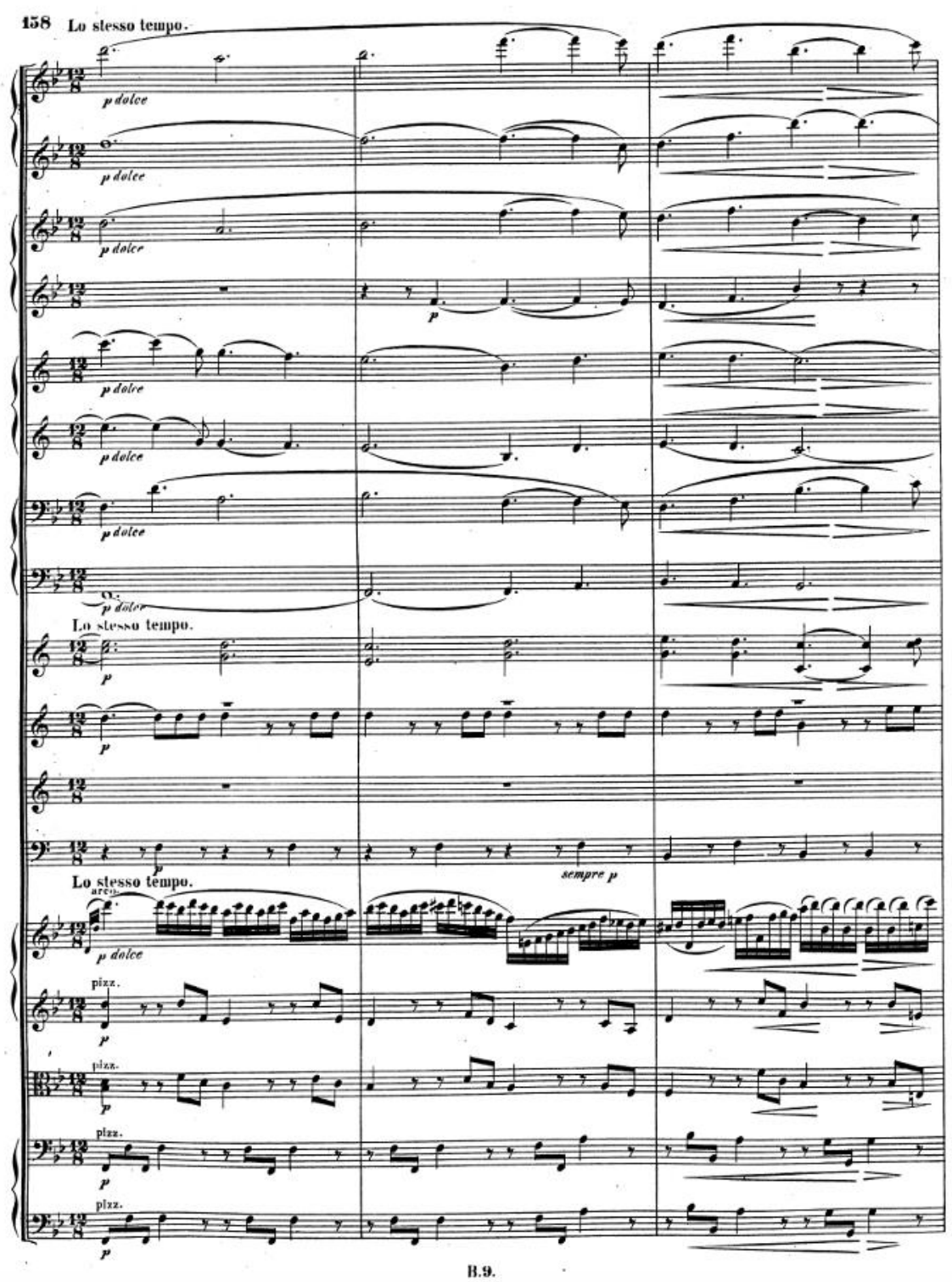

Fig. 3: Beethoven, Novena Sinfonia Op. 125, III 4

(imagen procedente de imslp.org).

\section{CONCLUSIONES}

En suma, el trabajo previo del director de orquesta y, por ende, el estudio de la dirección de orquesta desde el punto de vista obra se constituyen como elementos fundamentales en la comprensión de dicha labor. Incluso en el caso de los directores más prestigiosos, la obra se revela como el punto central de cualquier intento de dirección musical:

\footnotetext{
${ }^{4}$ Nótese la complejidad del discurso musical mencionado por Bernstein.
} 
[Zubin Mehta] Ha grabado las primeras cinco sinfonías de Mahler con DECCA, pero en 1980-1981 reconoció que todavía no se había atrevido con las últimas cuatro porque creía que no había acabado de entenderlas. En primer lugar, las había interpretado en muy pocas ocasiones, y nunca graba una pieza sin haberla interpretado en varias ocasiones, «porque cada vez nos revela más elementos, nuevos enfoques, detalles que se nos escaparon la primera vez; ni siquiera después de haberla escuchado doce veces se llega entrañar su completo significado. No he alcanzado ni de lejos ese nivel con las últimas sinfonías de Mahlè» (...) «La Octava [de Mahler] es, en cierto modo, la más fácil de dirigir, la más sencilla de interpretar, y, la más difícil, sin duda, la Séptima. Sólo la dirigí una vez; fue en Los Ángeles y no habría podido hacerlo sin la partitura y el análisis que me proporcionó Swarowsky, que no era otro que el de Webern, que Swarowsky conservaba con un recuerdo. Esta sinfonía me sigue intimidando. Es la más difícil que he dirigido en toda mi vida» (Matheopoulos, 2004, p. 264).

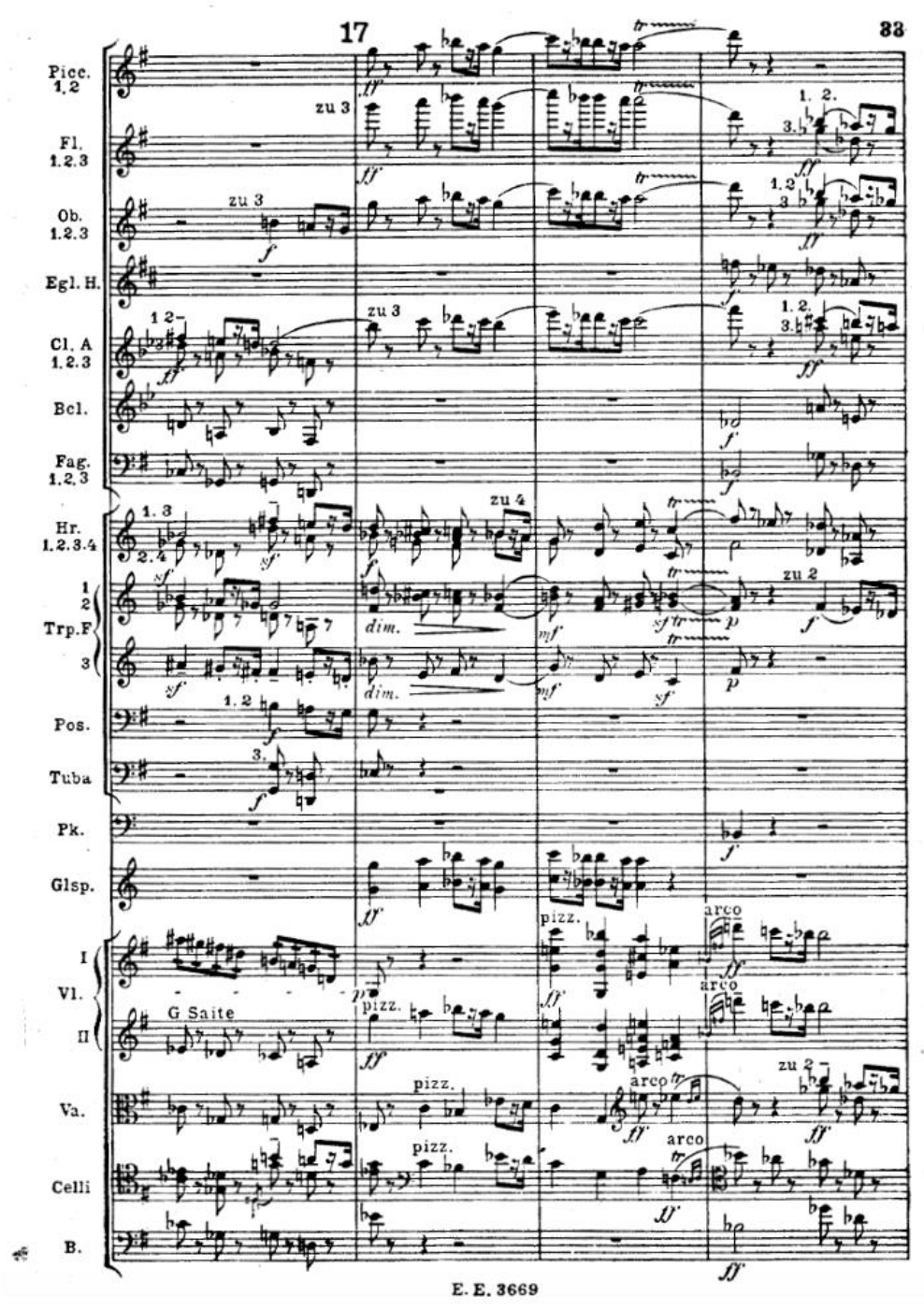

Fig. 4: Mahler, Séptima Sinfonía, I.

(imagen procedente de imslp.org). 


\section{MARTA VELA GONZÁLEZ}

En la actualidad, hasta la más moderna ciencia de tipo deportivo ha confirmado la utilidad de la práctica mental para la preparación y perfeccionamiento de cualquier disciplina psicomotriz, por tanto, también la música, en su perspectiva física, en cuanto a la imaginación del sonido y su generación a partir del gesto del director, puede aplicarse este método a su proceso de estudio.

Los deportistas, capaces de medir sus resultados con una precisión mucho mayor que la de los músicos, han sacado hace mucho tiempo partido «mensurable» de esta técnica. Ningún deportista puede de hoy renunciar ya a este método. Un esquiador que desciende aprende mentalmente y de memoria su camino. Si no lo dominan mentalmente, sus reacciones corporales tienen lugar demasiado tarde. Se vería obligado a rehacer en cada momento la constelación de sus movimientos corporales sobre la base de las nuevas impresiones sensoriales (Mantel, 2010, p. 226).

Por consiguiente, el análisis y su continua profundización sobre la obra musical se revela como herramienta fundamental en el proceso de estudio y en la capacidad del intérprete, entrenada por el tiempo, de conocer la materia sonora que está destinado a expresar bajo sus propias coordenadas artísticas, de ahí que la labor del director sea, en realidad -o debería ser-, la de cualquier músico, a partir de la imagen previa del sonido, forjada mediante la reflexión, la imaginación en la escucha, etc.

Sólo así lograremos hacer justicia a la obra musical confiada desde el pasado por los compositores para la pervivencia de nuestro arte.

\section{BIBLIOGRAFÍA}

Adorno, T. W. (2008). Monografías musicales. Madrid: Akal.

Ansermet, E. (2000). Escritos sobre música. Barcelona: Idea Música.

Brendel, A. (2013). De la A a la Z de un pianista. Barcelona: Acantilado.

Busoni, F. (2004). Pensamiento musical. Universidad Autónoma de México: México D.F.

Casella, A. (1936). El piano. Buenos Aires: Ricordi.

Deschausées, M. (2009). El intérprete y la música. Madrid: Rialp.

Furtwängler, W. (2012). Sonido y palabra. Barcelona: Acantilado.

Rink, J., (ed.) La interpretación musical, Madrid: Alianza.

Leimer, K. (1951). Rítmica, dinámica, pedal. Buenos Aires: Ricordi.

Leimer. K. (2007). La moderna ejecución pianistica. Buenos Aires: Ricordi. 


\section{APROXIMACIÓN METODOLÓGICA AL ESTUDIO DE LA DIRECCIÓN DE ORQUESTA}

DESDE EL PUNTO DE VISTA DE LA OBRA MUSICAL

Llort Llopart, V. (2005). Berlioz escritor. Barcelona: Tizona.

Mantel, G. (2010). Interpretación. Del texto al sonido. Madrid: Alianza.

Madigan, A. (2008). Un acto de libertad: charlando con Antoni Ros-Marbà. Barcelona: Ediciones Autor.

Matheopoulos, H. (2004). Maestro. Barcelona: Ediciones Robinbook.

Neuhaus, H. (1987). El arte del piano. Madrid: Real Musical.

Previtali, F. (2007). Guia para el estudio de la dirección orquestal. Buenos Aires: Melos.

Riemann, H. (2005). Manual del pianista. Cornellà de Llobregat: Idea Books.

Rosen, C. (2005). Las sonatas para piano de Beethoven. Madrid: Alianza.

Rosen, C., (2014). Elpiano: notas y vivencias. Madrid: Alianza.

Scherchen, H. (2005). El arte de dirigir la orquesta. Cornellà de Llobregat: Idea Books.

Schoenberg, A. (1943). Modelos para estudiantes de composición. Buenos Aires: Ricordi.

Schoenberg, A. (1974). Tratado de Armonía. Barcelona: Labor.

Schoenberg, A. (1990). Funciones estructurales de la armonía. Barcelona: Labor.

Schoenberg, A. (2007). El estilo y la idea. Alcorcón: Mundimúsica.

Stravinsky, I. (2006). Poética musical. Barcelona: Acantilado.

Swarowsky, H. (1989). Defensa de la obra. Madrid: Real Musical.

Thielemann, C. (2013). Mi vida con Wagner. Madrid: Akal.

Vela, M. (2017). La noción de paradigma en el estilo musical. Sinfonía Virtual, vol. 32.

Vela, M. (2018). Códigos simbólicos de la obra musical: influencia sobre la construcción de la interpretación instrumental. Revista musical de Venezuela, (55), pp. 94-118.

Walter, B. (2002). Gustav Mabler. Madrid: Alianza.

Walker, A. (1962). A study in musical analysis. London: Barrie and Rockliff.

Weingartner, F. (1911). Sur l'art de diriger. Leipzig: Breitkoff \& Härtel.

Fecha de recepción: 10/11/2020

Fecha de aceptación: 16/07/2021 